\title{
Energy consumption for shortcuts to adiabaticity
}

\author{
E. Torrontegui, ${ }^{1,2,{ }^{*}}$ I. Lizuain, ${ }^{3}$ S. González-Resines,${ }^{4}$ A. Tobalina, ${ }^{4}$ A. Ruschhaupt,${ }^{5}$ R. Kosloff, ${ }^{2}$ and J. G. Muga ${ }^{4, \dagger}$ \\ ${ }^{1}$ Instituto de Física Fundamental IFF-CSIC, Calle Serrano 113b, 28006 Madrid, Spain \\ ${ }^{2}$ Institute of Chemistry and The Fritz. Haber Research Center, The Hebrew University, Jerusalem 91904, Israel \\ ${ }^{3}$ Department of Applied Mathematics, University of the Basque Country UPV/EHU, Plaza Europa 1, 20018 Donostia-San Sebastian, Spain \\ ${ }^{4}$ Departamento de Química Física, Universidad del País Vasco-Euskal Herriko Unibertsitatea, Apartado 644, Bilbao, Spain \\ ${ }^{5}$ Department of Physics, University College Cork, Cork, Ireland
}

(Received 26 April 2017; published 25 August 2017)

\begin{abstract}
Shortcuts to adiabaticity let a system reach the results of a slow adiabatic process in a shorter time. We propose to quantify the "energy cost" of the shortcut by the energy consumption of the system enlarged by including the control device. A mechanical model where the dynamics of the system and control device can be explicitly described illustrates that a broad range of possible values for the consumption is possible, including zero (above the adiabatic energy increment) when friction is negligible and the energy given away as negative power is stored and reused by perfect regenerative braking.
\end{abstract}

DOI: 10.1103/PhysRevA.96.022133

\section{INTRODUCTION}

Shortcuts to adiabaticity (STAs) [1,2] are protocols for the time dependence of the control parameters of a system (hereafter primary system, PS) so that it reaches the same final conditions (energy, populations, or state) of a slow adiabatic process in a shorter time. STAs have found widespread applications in atomic, molecular, and optical physics and beyond, e.g., for classical systems [3-5], as a generic tool to combat decoherence and design robust, fast processes or devices. Some STAs use the structure of the Hamiltonian describing the slow process for the PS, as in invariant-based methods [1], and others add new control terms, as in counterdiabatic approaches [6], but this distinction does not affect the following discussion.

The total mechanical work done on the PS in a given STA is, by definition, equal to the work done in the adiabatic process, i.e., the adiabatic energy increment between initial and final states. It was soon clear that this quantity could not represent all relevant energy flows, which led to the consideration of alternative measures [7]. Several disparate definitions of energy cost have been proposed in the context of quantum thermodynamics to characterize quantum engines and refrigerators [8-18]. These definitions have been systematically formulated in terms of the cycling system (PS) alone. Even if the existing proposals have their own merits and applications, the point of view put forward in this article is that a broader perspective is necessary for the definition to be useful and practically relevant, addressing not only the PS but also the control system (CS) that drives the time-dependent parameters. In other words, we advocate redefining and expanding the "system" in the model to include the PS and the CS in an enlarged system. It might appear that this simply shifts the system-defining border so that the same problem is translated towards the new border. The important point is to find a meaningful divide, for which the energy changes with the outer world are modeled by forces that can be easily translated into fuel or electric power consumption by an active device.

\footnotetext{
*eriktm@iff.csic.es

†jg.muga@ehu.es
}

Such a shift is crucial to make the energy "cost" a significant quantity that indeed has something to do with the feasibility of the processes, minimal times allowed, or economic costs. Some examples help to clarify this: If a train (CS) transports cargo (PS) horizontally between two stations, the total energy increment of the cargo is zero. Surely what interests us more as a relevant cost is the energy consumption by the active force that the engines should do, translated into fuel consumption. We thus need to evaluate this force by expanding the physical model to include the train itself, taking into account friction and the braking mechanism and paying attention to the maximum power deliverable by the engine, which will put limits on the minimal transport times. Similar examples can be drawn from studies by nutritionists or biomechanicists concerned with the kilocalories the body consumes or the oxygen intake to perform a given task or exercise [19]. For a weightlifter (CS) pushing a weight (PS) up, the energy expenditure depends not only on the work done on the weight but also on CS-dependent factors such as the lifter's skill and weight and muscular mass.

This paper is based on a simple model for which enlightening, explicit expressions for the dynamics, power, and energy consumption are worked out. In Sec. II we present our model, a mechanical crane, and the main results. The model is described by equations similar to the ones used for the transport of neutral atoms or ions in microscopic traps. In Sec. III we find the optimal protocol with respect to energy consumption, and the paper ends with a discussion in which we surmise the implications that we expect to be broadly applicable.

\section{MODEL AND RESULTS}

The model is an overhead crane, as depicted in Fig. 1, composed of a trolley of mass $M$ (CS) moving along a horizontal bridge and a load of mass $m$ (PS) pending by a constant-length rope [20]. We neglect the stiffness and mass of the rope and air resistance. The load can be regarded, in the small-oscillation regime characteristic of these devices, as a harmonic oscillator with a moving center. The generalized coordinates are the position of the trolley $x(t)$ and the swing angle $\theta(t)$. The process we consider is a transport of the load by moving the trolley from $x=0$ to $x=d$ in a time $t_{f}$. If 


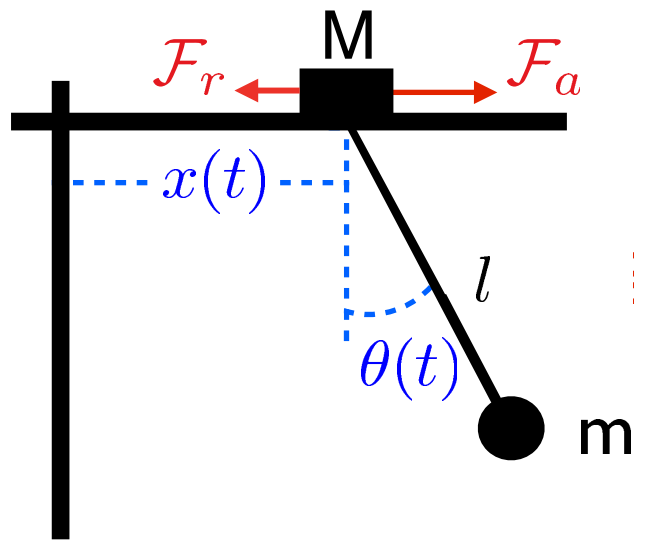

FIG. 1. Overhead crane composed of a load of mass $m$ and a trolley of mass $M$ connected through a rope of constant length $l$. The red solid arrows represent the active force $\mathcal{F}_{a}$ and the friction force $\mathcal{F}_{r}$ acting on a rightward-moving trolley.

done adiabatically, the initial and final energies of the load should be equal. Shortcuts for quantum systems subjected to a moving harmonic (or otherwise) trap have been extensively studied (see, e.g., [21-23]).

The external forces depicted in Fig. 1 are the actuating force $\mathcal{F}_{a}$ (e.g., due to an engine or to a braking mechanism if it opposes the direction of motion of the trolley) and the friction, modeled here as $\mathcal{F}_{r}=-\gamma \dot{x}, \gamma \geqslant 0$. The Lagrangian, without friction, is $\mathcal{L}=\mathcal{L}_{1}+\mathcal{L}_{2}$,

$$
\begin{aligned}
& \mathcal{L}_{1}=\frac{m}{2}\left[\dot{x}^{2}+l^{2} \dot{\theta}^{2}+2 l \dot{x} \dot{\theta} \cos \theta\right]+m g l \cos \theta, \\
& \mathcal{L}_{2}=\frac{M}{2} \dot{x}^{2}+\mathcal{F}_{a} x,
\end{aligned}
$$

where the dots represent time derivatives, $l$ is the rope length, and $g$ is the gravitational acceleration. With friction, the equations of motion are derived from the Euler-Lagrange equations, with the equation on the trolley position modified to include a friction term, $\frac{d}{d t}\left(\frac{\partial L}{\partial \dot{x}}\right)-\frac{\partial L}{\partial x}+\frac{\partial \mathcal{F}}{\partial \dot{x}}=0$, where $\mathcal{F}=\gamma \dot{x}^{2} / 2$ is Rayleigh's dissipation function [24],

$$
\begin{aligned}
0 & =l \ddot{\theta}+\ddot{x} \cos \theta+g \sin \theta, \\
\mathcal{F}_{a}+\mathcal{F}_{r} & =M \ddot{x}+m\left(\ddot{x}+l \ddot{\theta} \cos \theta-l \dot{\theta}^{2} \sin \theta\right) .
\end{aligned}
$$

Equation (2) defines the kinematics of the load in terms of only $x(t)$; that is, it is formally independent of characteristics of the trolley such as mass or friction for a given $x(t)$. This allows the formal treatment of the load as an open system subject to an external time-dependent control, but $x(t)$ depends on these characteristics and on the angle and the pulling force via Eq. (3). We may compute the frictionless Hamiltonian of the total system through the Lagrangian $\mathcal{L}=\mathcal{L}_{1}+\mathcal{L}_{2}$ given by Eq. (1), $\mathcal{H}=\dot{x} p_{x}+\dot{\theta} p_{\theta}-\mathcal{L}$, where $p_{x}=\partial \mathcal{L} / \partial \dot{x}$ and $p_{\theta}=$ $\partial \mathcal{L} / \partial \dot{\theta}$. To account for friction, one of Hamilton's equations changes to $[25,26] \dot{p}_{x}=-\frac{\partial \mathcal{H}}{\partial x}-\frac{\partial \mathcal{F}}{\partial \dot{x}}$. The power produced by the force $\mathcal{F}_{a}$ can be expressed as the rate of change of $\mathcal{H}_{0}=$ $\mathcal{H}+\mathcal{F}_{a} x$ (the last term cancels the external interaction $-\mathcal{F}_{a} x$ in $\mathcal{H}$, leaving the bare mechanical energy) plus the energy loss rate due to friction,

$$
\mathcal{P}=\frac{d \mathcal{H}_{0}}{d t}+\gamma \dot{x}^{2}=\mathcal{F}_{a} \dot{x}
$$

The total derivative is computed along the trajectory making use of Hamilton's equations for $\mathcal{H}$ modified by the friction term. Here a meaningful divide is established, with the relevant connection to the outer world being a force $\mathcal{F}_{a}$ produced by an external engine that, for positive power, consumes fuel to increase the internal mechanical energy and fight against friction. The total energy consumption could be defined as the integral of the power [20], but this would ignore the peculiarities of braking phases where $\mathcal{F}_{a}$ and $\dot{x}$ have different signs. We propose instead a more realistic expression parameterized by $-1 \leqslant \eta \leqslant 1$, which depends on the braking mechanism,

$$
\mathcal{E}=\int_{0}^{t_{f}} d t \mathcal{P}_{+}+\eta \int_{0}^{t_{f}} d t \mathcal{P}_{-}=\mathcal{E}_{+}+\eta \mathcal{E}_{-},
$$

where $\mathcal{P}_{ \pm}=\Theta( \pm \mathcal{P}) \mathcal{P}$ are the positive and negative parts of $\mathcal{P}$ for accelerating or braking phases of the trolley motion and $\Theta$ is the Heaviside function. $\mathcal{E}_{ \pm}$are the positive and negative parts of the integral. While more sophisticated descriptions are possible, with $\eta$ depending on several variables, our aim here is to set a crude model that captures the essence of the energy trade during braking and provides limiting scenarios: $\eta=1$ corresponds to a mechanism able to fully accumulate the braking energy $\mathcal{E}_{-}$and give it back on demand, i.e., perfect regenerative breaking; $\eta=-1$ corresponds to using the engine in both phases of the motion, whereas $\eta=0$ is the limit in which braking fully dissipates the energy loss of the system with negligible energy consumption.

To find STAs we use the horizontal deviation of the load from the trolley position, $q(t)=l \sin \theta(t)$, and assume the small-oscillation regime. Equation (2) becomes

$$
\ddot{q}+\omega^{2} q=-\ddot{x},
$$

where $\omega^{2}=g / l$. The dynamics of the load (PS) is described in a moving frame by a forced harmonic oscillator, which can be derived from the Hamiltonian

$$
H=\frac{p^{2}}{2 m}+\frac{1}{2} m \omega^{2} q^{2}+m \ddot{x} q,
$$

where $p=m \dot{q}$ is the canonical momentum of $q$. Associated with $H$ is an invariant of motion [27]

$$
I=\frac{1}{2 m}(p-m \dot{\alpha})^{2}+\frac{m}{2} \omega^{2}(q-\alpha)^{2},
$$

where $\alpha(t)$ is an auxiliary trajectory that must follow the dynamics of a forced harmonic oscillator [27],

$$
\ddot{\alpha}+\omega^{2} \alpha=-\ddot{x} .
$$

We choose $\alpha(t)$ functions that satisfy the boundary conditions (BCs) $\alpha\left(t_{b}\right)=\dot{\alpha}\left(t_{b}\right)=\ddot{\alpha}\left(t_{b}\right)=0$ for $t_{b}=0, t_{f}$. In this way $\ddot{x}\left(t_{b}\right)=0$ and, from Eqs. (7) and (8), $H(0)=I(0)=E_{0}$ for any arbitrary trajectory $q(t)$ satisfying Eq. (6) with initial energy $E_{0}$ (for the auxiliary trajectory $\alpha, E_{0}=0$ ). As $I$ is invariant, $I\left(t_{f}\right)=E_{0}$. Moreover, the final energy is $H\left(t_{f}\right)=$ $I\left(t_{f}\right)=E_{f}$. In summary, imposing the appropriate BCs on $\alpha, E_{f}=E_{0}$ for any trajectory, as for an adiabatic, slow process, but in a finite time. 

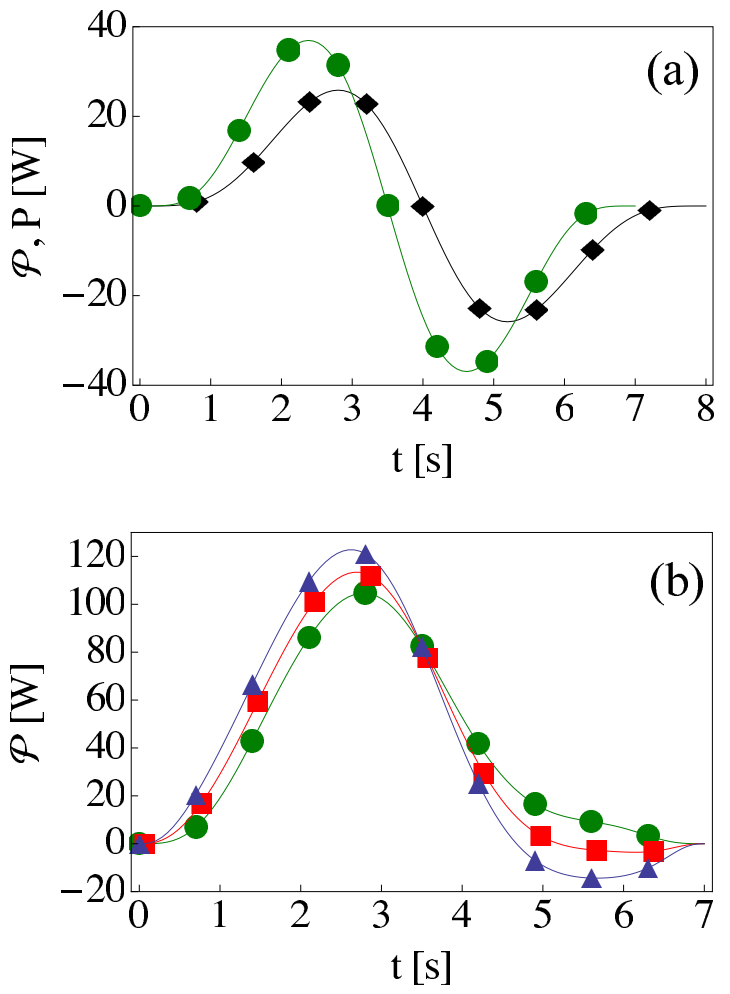

FIG. 2. The total power $\mathcal{P}$ to control $x(t)$ for different $M$ and friction coefficients and load power $P$. Symbols represent $\mathcal{P}$ using the small-oscillation approximation, Eq. (10), and lines represent using the exact Eq. (4). (a) Power $P$ of the load (symbols) and total power $\mathcal{P}$ (lines) in the $M=\gamma=0$ limit for $t_{f}=7 \mathrm{~s}$ (green line and circles) and $t_{f}=8 \mathrm{~s}$ (black line and diamonds). (b) Total power $\mathcal{P}$ with friction, $\gamma=15 \mathrm{~kg} / \mathrm{s}, t_{f}=7 \mathrm{~s}$, for different values of the trolley mass: $M=0 \mathrm{~kg}$ (green solid line and circles), $M=10 \mathrm{~kg}$ (red solid line and squares), and $M=20 \mathrm{~kg}$ (blue solid line and triangles). $m=10 \mathrm{~kg}, l=5 \mathrm{~m}, d=10 \mathrm{~m}, q(0)=0 \mathrm{~m}, \dot{q}(0)=0 \mathrm{~m} / \mathrm{s}$, and $g=9.8 \mathrm{~m} / \mathrm{s}^{2}$.

We interpolate $\alpha(t)$ with a polynomial, $\alpha(t)=\sum_{i=0}^{7} a_{i} t^{i}$, where the first six coefficients $\left(a_{0}-a_{5}\right)$ are derived from the six BCs for $\alpha$. The trajectory $x(t)$ of the trolley is deduced from Eq. (9), $x(t)=-\int_{0}^{t} d t^{\prime} \int_{0}^{t^{\prime}} d t^{\prime \prime}\left[\ddot{\alpha}\left(t^{\prime \prime}\right)+\omega^{2} \alpha\left(t^{\prime \prime}\right)\right]$ and satisfies $\ddot{x}\left(t_{b}\right)=\dot{x}(0)=x(0)=0$. The coefficients $a_{6}$ and $a_{7}$ are set by demanding $\dot{x}\left(t_{f}\right)=0$ and $x\left(t_{f}\right)=d$. Due to the freedom to design $\alpha$, optimal-control theory could be used to find trolley trajectories that optimize a chosen variable given some physical constraints [21]. For small oscillations, the total power in Eq. (4) takes the form

$$
\mathcal{P}=\left(M \ddot{x}-m q \omega^{2}+\gamma \dot{x}\right) \dot{x},
$$

plotted in Fig. 2 for $q(t)=\alpha(t)$. The terms in parentheses represent the force to move a free trolley (with no load or friction) minus the force that the load exerts on the trolley (a "pull or drag" back-action whose sign depends on their relative positions) minus the friction force (which always gives a positive contribution to the power). Let us compare this quantity to the power on the load, $P=\frac{d E(t)}{d t}$, where $E(t)$ is the mechanical energy of the load, $E(t)=m(\dot{x}+\dot{q})^{2} / 2+$ $m \omega^{2} q^{2} / 2$. [For arbitrary $t$, this is different from $H(t)$ since
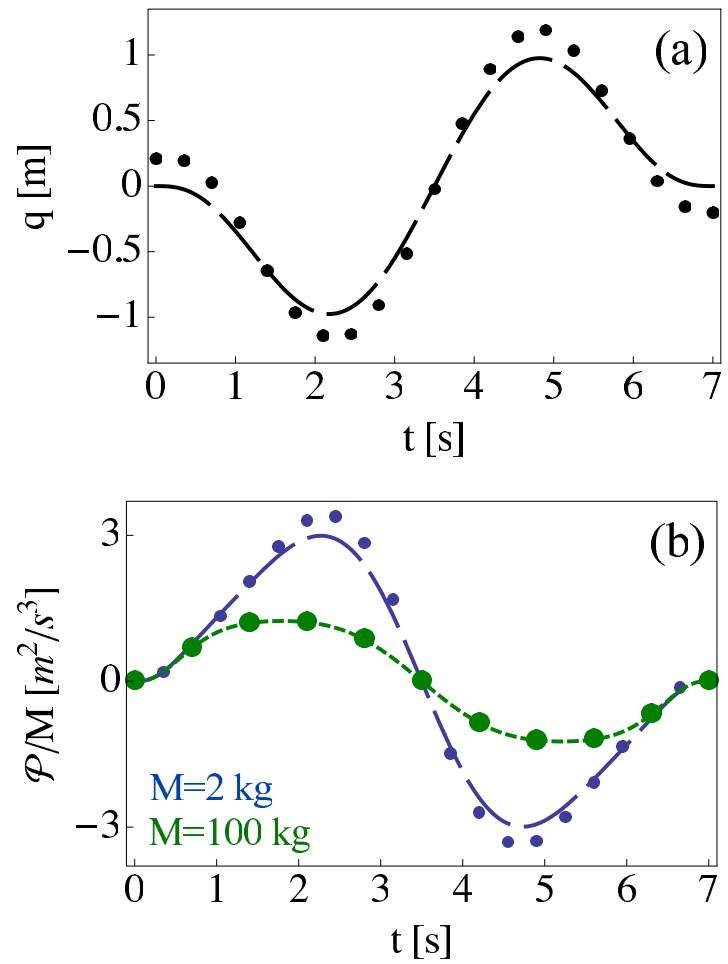

FIG. 3. Effect of the trajectory $q(t)$ on the total power $\mathcal{P}$ for different trolley masses. $q(0)=\dot{q}(0)=0$ (dashed lines), $q(0)=$ $0.2 \mathrm{~m}$, and $\dot{q}(0)=0.1 \mathrm{~m} / \mathrm{s}$ (circles). (a) $q(t)$ for different initial conditions. (b) Corresponding power consumed for different $m / M$ ratios: $M=2 \mathrm{~kg}$ (blue long-dashed line and small circles) and $M=100 \mathrm{~kg}$ (green short-dashed line and big circles). $m=1 \mathrm{~kg}$, $l=5 \mathrm{~m}, d=10 \mathrm{~m}, t_{f}=7 \mathrm{~s}, \gamma=0 \mathrm{~kg} / \mathrm{s}$, and $g=9.8 \mathrm{~m} / \mathrm{s}^{2}$.

$H$ is defined in a moving frame, but they coincide at the boundary times.] Using Eq. (6), $P=-m q \omega^{2} \dot{x}$, which is the rate of energy change in the PS, but for a given $x(t)$, it ignores other features of the trolley. In contrast, $\mathcal{P}$ and $\mathcal{E}$ generally depend [see Eq. (10)] on the characteristics of the CS $(M, \gamma)$, on its dynamics $(\dot{x}, \ddot{x})$, and on the deviation of the load $q(t)$. If $M=\gamma=0, \mathcal{P}_{M=\gamma=0}=-m q \omega^{2} \dot{x}=P$ (see Fig. 2). A practical advantage of the limit $M \gg m$ is that $\mathcal{P}$ can be made essentially independent of $q(t)$, i.e., of the initial conditions $\{q(0), \dot{q}(0)\}$ (see Fig. 3), where the $\alpha(t)$ chosen implies that $\ddot{x}=0$ at the boundary times and at the middle time. This stabilization comes with a price, namely, higher-power peaks due to a larger $M$.

The integral of $\mathcal{P}$, without friction, $\gamma=0$, is zero by construction of the STAs (the final adiabatic energy of the load must be equal to the initial one, and the trolley starts and ends at rest), so the total energy consumption would be zero for $\eta=1$. The other parameters may be arbitrary, even $t_{f}$, within small oscillations. Friction and realistic braking mechanisms $(\eta \neq 1)$ imply $\left|\eta \mathcal{E}_{-}\right|<\mathcal{E}_{+}$and therefore dependences of $\mathcal{E}>0$ on $\gamma, M$, or $t_{f}$. Note that $\mathcal{E}$ depends linearly on $\eta$ with minimum $\mathcal{E}_{+}+\mathcal{E}_{-}$at $\eta=1$ and maximum $\mathcal{E}_{+}-\mathcal{E}_{-}$at $\eta=-1$. Since the time integral of the frictionless part of Eq. (10) is zero, we get, using the Euler-Lagrange equation, the lower bound

$$
\mathcal{E} \geqslant \gamma d^{2} / t_{f},
$$



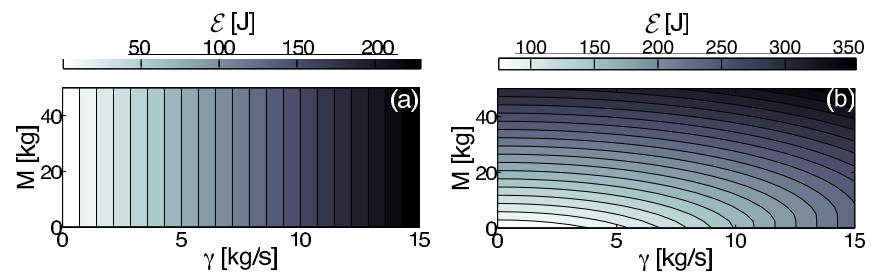

FIG. 4. Contour surface of the energy consumption $\mathcal{E}$ as a function of the CS variables $M$ and $\gamma$ for different values of the $\eta$ parameter: (a) $\eta=1$ and (b) $\eta=-1 . m=10 \mathrm{~kg}, l=5 \mathrm{~m}, d=10 \mathrm{~m}$, $t_{f}=9 \mathrm{~s}, q(0)=0 \mathrm{~m}, \dot{q}(0)=0 \mathrm{~m} / \mathrm{s}$, and $g=9.8 \mathrm{~m} / \mathrm{s}^{2}$.

which is valid for all $\eta$. This agrees with Landauer's expectation for energy costs of processes not involving information losses [28]. However, a different, tighter bound is found in Sec. III from the optimal protocol.

Some trends are seen in Figs. 2 and 4: Friction enhances $\mathcal{E}_{+}$ and diminishes or even suppresses $\mathcal{E}_{-}$. A larger $M$ generally increases the power peaks and also hinders the suppression of $\mathcal{E}_{-}$by friction; longer process times decrease power peaks and, typically, $\mathcal{E}$ too, with the mentioned exception of an ideal setting, $\gamma=0, \eta=1$, for which $\mathcal{E}=0$ for any time $t_{f}$. The contour plots of $\mathcal{E}$ for $\eta= \pm 1$ are quite different (see Fig. 4), with $\mathcal{E}$ independent of $M$ if $\eta=1$ and nearly independent of $\gamma$ for weak friction if $\eta=-1$.

The feasibility of a given STA will depend not only on the additive energy consumption $\mathcal{E}$ but also on the possibility to deliver the instantaneous power peaks, which increase with diminishing process times. STAs can be designed to lower the peak in $\mathcal{P}$, as done for $P$ in [29]. The mean-value theorem provides bounds for the peak of $\mathcal{P}$ in different regimes dominated by one of the terms in Eq. (10): $\mathcal{P} \geqslant M d^{2} / t_{f}^{3}$ for a regime dominated by the trolley frictionless dynamics ( $M$ term), whereas $\mathcal{P} \geqslant \gamma d^{2} / t_{f}^{2}$ for a friction-dominated one. Finally, peak bounds for $M=\gamma=0$ scale as $m d^{2} / t_{f}^{3}$ at long process times and as $4 m d^{2} /\left(\omega^{2} t_{f}^{5}\right)$ at short times. (The bounds at short times are meaningful only for a pure harmonic oscillator since the pendulum will abandon the small-oscillation regime, and we have assumed $\sqrt{2 E_{0} / m} / \omega \ll d$.) Minimal times for a given maximal power can be read directly from the bounds.

\section{PROTOCOL FOR MINIMAL ENERGY CONSUMPTION}

We use the degeneracy of the STAs to design a protocol that minimizes energy consumption, combining inverseengineering STAs with optimal-control theory [21,30]. In this section we assume that the harmonic model holds.

It is convenient to use the horizontal position of the load in the laboratory frame, $X \equiv q+x$, which obeys the Newton equation

$$
\ddot{X}+\omega^{2}(X-x)=0 .
$$

Like we did for the difference between a general $q$ and a particular trajectory $\alpha$ in the previous section, we distinguish a particular trajectory $\xi$ that satisfies Eq. (12) and the boundary conditions $\xi(0)=0, \xi\left(t_{f}\right)=d$ and $\dot{\xi}\left(t_{b}\right)=\ddot{\xi}\left(t_{b}\right)=0$, with $t_{b}=0, t_{f}$. To follow the usual conventions in optimal-control theory, we use a new notation,

$$
y_{1}=\xi, \quad y_{2}=\dot{\xi}, \quad u(t)=x,
$$

where $y_{1}, y_{2}$ are the components of a "state vector" $\mathbf{y}$ and the trolley position $u(t)$ is considered the (scalar) control function. With this notation Eq. (12) for $\xi$ becomes

$$
\begin{aligned}
& \dot{y}_{1}=y_{2}, \\
& \dot{y}_{2}=-\omega^{2}\left(y_{1}-u\right) .
\end{aligned}
$$

The optimal-control problem is to find $|u(t)| \leqslant \delta$ for some fixed bound $\delta$, with $u(0)=0$ and $u\left(t_{f}\right)=d$, such that the system starts at $\left\{y_{1}(0)=0, y_{2}(0)=0\right\}$, ends up at $\left\{y_{1}\left(t_{f}\right)=\right.$ $\left.d, y_{2}\left(t_{f}\right)=0\right\}$, and minimizes a cost function $J$.

In order to match the boundary conditions at the initial and final times, the optimal control obtained may be complemented by appropriate jumps. We use Pontryagin's maximum principle, which provides necessary conditions for optimality [31]. Generally, to minimize the cost function

$$
J(u)=\int_{0}^{t_{f}} g(\mathbf{y}(t), u) d t,
$$

the maximum principle states that for the dynamical system $\dot{\mathbf{y}}=\mathbf{f}(\mathbf{y}(t), u)$, the coordinates of the extremal vector $\mathbf{y}(t)$ and of the corresponding adjoint state $\mathbf{k}(t)$ formed by Lagrange multipliers $k_{1}, k_{2}$ fulfill the Hamilton's equations for a control Hamiltonian $H_{c}$,

$$
\begin{aligned}
\dot{\mathbf{y}} & =\frac{\partial H_{c}}{\partial \mathbf{k}}, \\
\dot{\mathbf{k}} & =-\frac{\partial H_{c}}{\partial \mathbf{y}},
\end{aligned}
$$

where $H_{c}$ is defined as

$$
H_{c}(\mathbf{k}(t), \mathbf{y}(t), u)=k_{0} g(\mathbf{y}(t), u)+\mathbf{k}^{T} \cdot \mathbf{f}(\mathbf{y}(t), u) .
$$

The superscript $T$ used here denotes the transpose of a vector, and $k_{0}<0$ can be chosen for convenience since it amounts to multiplying the cost function by a constant. The (augmented) vector with components $\left(k_{0}, k_{1}, k_{2}\right)$ is nonzero and continuous. Note that the Lagrange multiplier $k_{0}$ is a constant; however, $k_{1}$ and $k_{2}$ are time dependent since the equations of motion (14) and (15) must be satisfied at all times. For almost all $0 \leqslant t \leqslant t_{f}$ the function $H_{c}(\mathbf{k}(t), \mathbf{y}(t), u)$ attains its maximum at $u=u^{*}$, and $H_{c}\left(\mathbf{k}(t), \mathbf{y}(t), u^{*}\right)=c$, where $c$ is constant. Assuming that the integrals of two of the terms of the total power (10) depending on $M$ and $m$ vanish (this is explicitly confirmed later), we shall consider only the term $\gamma \dot{x}^{2}$, so the cost function is

$$
J_{\mathcal{P}}=\int_{0}^{t_{f}} \dot{x}^{2} d t=\int_{0}^{t_{f}} \dot{u}^{2} d t
$$

for an "unbounded problem" (i.e., without restrictions on the possible values of the control) and an ideal $(\eta=1)$ type of process with perfect regenerative braking. The control Hamiltonian is

$$
H_{c}\left(k_{1}, k_{2}, y_{1}, y_{2}, u\right)=k_{0} \dot{u}^{2}+k_{1} y_{2}-k_{2} \omega^{2}\left(y_{1}-u\right),
$$

which sets the costate equations

$$
\dot{k}_{1}=\omega^{2} k_{2}, \dot{k}_{2}=-k_{1} \text {. }
$$



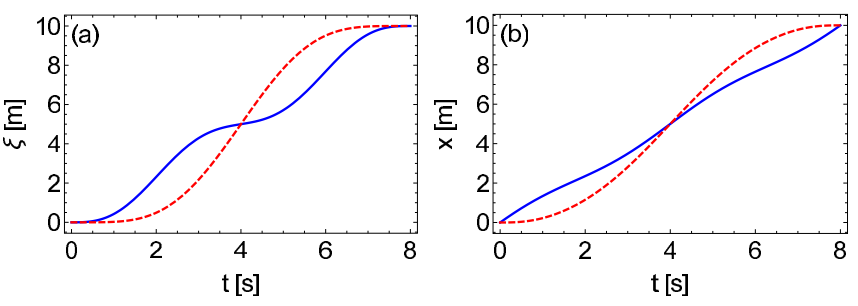

FIG. 5. (a) Designed function $\xi=\alpha+x$ as a function of time. Polynomial interpolation used in Sec. II (red dashed line) and optimal solution to minimize the energy consumption (blue solid line). (b) Trolley displacement $x$ as a function of time. Parameter values: $t_{f}=8 \mathrm{~s}, l=5 \mathrm{~m}, d=10 \mathrm{~m}, g=9.8 \mathrm{~m} / \mathrm{s}^{2}$, and $k_{0}=-1$.

The solution to this set of equations is

$$
\begin{aligned}
& k_{1}(t)=c_{1} \cos (\omega t)+\omega c_{2} \sin (\omega t), \\
& k_{2}(t)=c_{2} \cos (\omega t)-\frac{c_{1}}{\omega} \sin (\omega t),
\end{aligned}
$$

where $c_{1}$ and $c_{2}$ are arbitrary constants. According to Pontryagin's maximum principle, the time-optimal control $u(t)$ maximizes the control Hamiltonian $H_{c}$. Using the EulerLagrange equation, this is done when $u$ satisfies $k_{2} \omega^{2}=2 k_{0} \ddot{u}$. Using Eq. (23), we find

$$
u(t)=x(t)=c_{3}+t c_{4}-\frac{c_{2}}{2 k_{0}} \cos (\omega t)+\frac{c_{1}}{2 k_{0} \omega} \sin (\omega t),
$$

with $c_{3}$ and $c_{4}$ also being arbitrary constants. Finally, solving the differential equation (12), the optimal $\xi(t)$ is found. The constants are fixed by imposing the boundary conditions on $\xi$. In Fig. 5(a) we plot the optimal function $\xi$ and the one deduced from Sec. II with a polynomial $\alpha$. The optimal trolley displacement $x_{\mathrm{op}}(t)$ [Fig. 5(b)] satisfies $x_{\mathrm{op}}(0)=0$ and $x_{\mathrm{op}}\left(t_{f}\right)=d$,

$$
\begin{aligned}
x_{\mathrm{op}}(t)= & {\left[d \left\{-2+\omega^{2} t_{f} t+2 \cos (\omega t)-2 \cos \left[\omega\left(t-t_{f}\right)\right]\right.\right.} \\
& +2 \bar{c}+\omega t \bar{s}\}] /\left[-4+t_{f}^{2} \omega^{2}+4 \bar{c}+\omega t_{f} \bar{s}\right],
\end{aligned}
$$

with $\bar{c}=\cos \left(\omega t_{f}\right)$ and $\bar{s}=\sin \left(\omega t_{f}\right)$. However, $\dot{x}_{\mathrm{op}}\left(0^{+}\right)=$ $\dot{x}_{\mathrm{op}}\left(t_{f}^{-}\right) \neq 0, \ddot{x}_{\mathrm{op}}\left(0^{+}\right)=-\ddot{x}_{\mathrm{op}}\left(t_{f}^{-}\right) \neq 0$, and instantaneous jumps are required to satisfy the boundary conditions $\dot{x}\left(0^{-}\right)=\dot{x}\left(t_{f}^{+}\right)=\ddot{x}\left(0^{-}\right)=\ddot{x}\left(t_{f}^{+}\right)=0$, where the plus (minus) represents an approach from the right (left). The trajectory (25) must be limited to the domain $0<t<t_{f}$ and must be complemented by $x_{\mathrm{op}}=0$ for $t<0$ and $x_{\mathrm{op}}=d$ for $t>t_{f}$. $\dot{x}$ is discontinuous at $t=0$ jumping from zero to $x_{\mathrm{op}}\left(0^{+}\right)$. Similarly, at $t_{f}, \dot{x}$ jumps from $\dot{x}\left(t_{f}^{-}\right)$to zero. The acceleration thus includes Dirac $\delta$ impulses [29,32],

$$
\ddot{x}_{\mathrm{op}}= \begin{cases}0, & t \leqslant 0^{-}, \\ \dot{x}_{\mathrm{op}}\left(0^{+}\right) \delta(t), & 0^{-}<t<0^{+}, \\ \ddot{x}_{\mathrm{op}}(t), & 0^{+} \leqslant t \leqslant t_{f}^{-}, \\ -\dot{x}_{\mathrm{op}}\left(t_{f}^{-}\right) \delta\left(t-t_{f}\right), & t_{f}^{-}<t<t_{f}^{+}, \\ 0, & t_{f}^{+} \leqslant t,\end{cases}
$$

where $\dot{x}_{\text {op }}$ and $\ddot{x}_{\text {op }}$ represent the first and second time derivatives of Eq. (25). This implies that $q, X$, and $\dot{X}$ are continuous at the edges. The protocol, including the jumps, is indeed a shortcut, as the mechanical energy of the load,
$E(t)=m(\dot{x}+\dot{q})^{2} / 2+m \omega^{2} q^{2} / 2$, is equal at initial $\left(0^{-}\right)$and final $\left(t_{f}^{+}\right)$times. This can be seen from the vanishing of the integral

$$
\int_{0^{-}}^{t_{f}^{+}} q \dot{x}_{\mathrm{op}} d t=0,
$$

which does not get any contribution at the edges, $E\left(0^{-}\right)=$ $E\left(0^{+}\right)=E\left(t_{f}^{-}\right)=E\left(t_{f}^{+}\right)$. Comparing explicitly load mechanical energies immediately before and after the boundary times, this is consistent with the following jumps in $\dot{q}$ :

$$
\begin{aligned}
& \dot{q}\left(0^{+}\right)=\dot{q}\left(0^{-}\right)-\dot{x}\left(0^{+}\right), \\
& \dot{q}\left(t_{f}^{+}\right)=\dot{q}\left(t_{f}^{-}\right)+\dot{x}\left(0^{-}\right) .
\end{aligned}
$$

The total mechanical energy,

$$
E_{\mathrm{tot}}(t)=E(t)+\frac{1}{2} M \dot{x}^{2},
$$

is also equal at initial and final times since the trolley begins and ends at rest,

$$
\int_{0^{-}}^{t_{f}^{+}} \ddot{x}_{\mathrm{op}} \dot{x}_{\mathrm{op}} d t=0 .
$$

In more detail, the integral vanishes in the interior domain, from $0^{+}$to $t_{f}^{-}$, since $\dot{x}_{\mathrm{op}}\left(0^{+}\right)=\dot{x}_{\mathrm{op}}\left(t_{f}^{-}\right)$, and the jumps due to initial and final $\delta$ impulses compensate, $\int_{0^{-}}^{0^{+}} M \ddot{x}_{\mathrm{op}} \dot{x}_{\mathrm{op}} d t=$ $M \dot{x}_{\mathrm{op}}^{2}\left(0^{+}\right) / 2$ and $\int_{t_{f}^{-}}^{t_{f}^{+}} M \ddot{x}_{\mathrm{op}} \dot{x}_{\mathrm{op}} d t=-M \dot{x}_{\mathrm{op}}^{2}\left(t_{f}^{-}\right) / 2$. Moreover, since the singularity of $\dot{x}_{\mathrm{op}}$ at the boundaries corresponds to a finite jump,

$$
\int_{0^{-}}^{0^{+}} \dot{x}_{\mathrm{op}}^{2} d t=0, \quad \int_{t_{f}^{-}}^{t_{f}^{+}} \dot{x}_{\mathrm{op}}^{2} d t=0,
$$

the Dirac impulses do not contribute to the energy dissipated by friction. Using expression (25) for the optimal trajectory, we find the explicit expression for the minimal energy consumption. This sets a bound for any other process,

$$
\mathcal{E} \geqslant \frac{\gamma d^{2}}{t_{f}+\frac{4\left[-1+\cos \left(\omega t_{f}\right)\right]}{\omega\left[\omega t_{f}+\sin \left(\omega t_{f}\right)\right]}},
$$

tighter than Eq. (11), $\mathcal{E} \geqslant \gamma d^{2} / t_{f}$. At large times, compared to the oscillation period, they coincide. Indeed, $\gamma d^{2} / t_{f}$ agrees with Landauer's prediction on the energy dissipation proportional to the "velocity of the process" when there is no information loss [28]. However, whereas he emphasized that the dissipation can be made arbitrarily small for sufficiently long times, STAs are, by construction, intended as fast processes where the dissipation due to friction does not vanish. A second difference with Landauer's discussion is that at short times, the dependence in Eq. (33) changes to

$$
\mathcal{E} \gtrsim \frac{720 d^{2}}{\omega^{4} t_{f}^{5}}
$$

with the caveat that this result indeed requires harmonic oscillator dynamics.

Note that the discontinuities in the derivatives of $x_{\mathrm{op}}(t)$ imply infinite-power peaks, but the energy consumed by the 
engine controlling the motion of the trolley, which is equal to the dissipated energy since the initial and final mechanical energies are equal, is finite. The ability to approach this ideal scenario of infinite-power peaks will depend on the characteristics of the engine, but in any case, the bound (33) sets the minimum energy required to produce a STA protocol for a given transport time $t_{f}$.

\section{DISCUSSION}

We have worked out an explicit model to analyze the energy consumption in shortcuts to adiabaticity. The model helps to point out a number of fundamental aspects, such as the importance of considering the control system together with the primary system. In our model the power for the primary system and the total power agree only in a rather unrealistic scenario, namely, a control system with zero mass and no friction. The small mass limit of the control system is not only unrealistic but also undesirable, as it would make the total power and the external actuating control force depend on the specific dynamics (i.e., the initial boundary conditions) of the primary system. This is against the spirit of useful shortcuts, intended to take systems from initial to final Hamiltonian configurations without final excitation, irrespective of the initial conditions. Control systems for microscopic primary systems will typically involve macroscopic masses, currents, or classical fields, so the need to consider the control system to examine energy costs will be prevalent.

The model also provides an ideal test bed to realize that different types of braking affect the results dramatically. It illustrates that the stability of a given control protocol with respect to the primary system dynamics implies an energy cost and higher-power peaks, and it underlines the importance of both integrated and local-in-time quantities to determine the feasibility of shortcuts.

The current analysis may be extended to further classical, quantum, or hybrid systems. In particular a quantum load represented by a particle in a harmonic trap could be driven by exactly the same STA protocols devised here since $I$ and $H$ have the same form as in our model. Close to the current model is the transport of ions or neutral atoms for which different experiments have been performed or are planned [33-35]. For the transport of ultracold atoms in [33], the trap was formed by optical tweezers, moved by displacing a lens mounted on a motorized translation stage. This setting realizes the stabilizing $M>m$ limit, a typical scenario with microscopic loads. Similarly, Zenesini et al. moved an optical lattice by displacing the mirror mounted on piezoelectric actuators [36]. For ion transport in linear, multielectrode Paul traps, the cost will involve assessing the energy consumed by the microchip controlling the effective moving trap by means of time-varying electrode potentials. The stabilization of the total power will depend on the macroscopic charges in the electrodes to change the voltages being much larger than the ion charge.

While the results so far have been for a harmonic potential, deviations from the harmonic approximation could be taken into account following [37]. We may also consider initial angles of the load $\theta_{i}$ beyond the small-oscillation regime and redesign the protocol for the trolley motion $x(t)$ to minimize the difference between initial and final mechanical energies

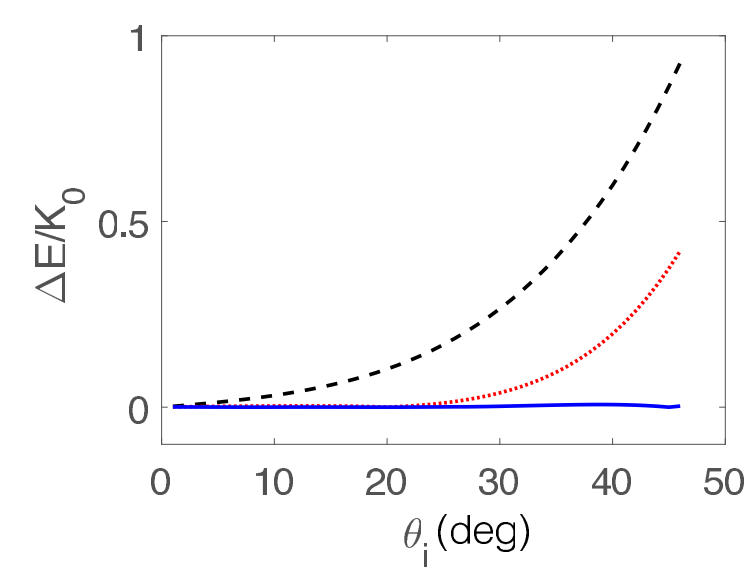

FIG. 6. Energy excitation of the load versus initial angle (with load initially at rest) in an inversely engineered transport process with $d=10 \mathrm{~m}, t_{f}=10 \mathrm{~s}, l=5 \mathrm{~m}$, and $m=10 \mathrm{~kg}$, using additional free parameters in the ansatz for $\alpha(t)$. The scaling factor is the kinetic energy for a constant-velocity process, $K_{0}=m d^{2} /\left(2 t_{f}^{2}\right)$. Black dashed line: process without additional parameters; red dotted line: one parameter added to minimize excitation in $\theta_{i}=20^{\circ}$ ( $\left.b_{8}=-3513.3\right)$; solid blue line: minimization for the excitation in $\theta_{i}=20^{\circ}$ and $\theta_{i}=45^{\circ}$ using two free parameters $\left(b_{8}=-13862\right.$ and $\left.b_{9}=2941.5\right)$.

of the load $\left(\Delta E=\left|E_{f}-E_{0}\right|\right)$. This requires a higher-order polynomial functions $\alpha(t)=\sum_{j=0}^{7+n} b_{j} t^{j}$ to minimize the energy difference for one or more $(n)$ initial angles $\theta_{i}$ with the extra parameters. The number of free parameters $n$ is set by the number of initial angles used to minimize the excitation, and the rest of the coefficients in $\alpha(t)$ are fixed by the boundary conditions as in Sec. II. In Fig. 6 we plot the excitation energy for processes with one and two free parameters and for the process in Sec. II $(n=0)$. Figure 6 demonstrates clearly that STAs beyond the small-oscillation regime are indeed possible. This implies zero or negligible energy consumption under ideal conditions (no friction, $\gamma=0$, and regenerative braking, $\eta=1)$.

For a general system, beyond transport systems, regardless of the specific dynamics involved, friction, the combination of positive and negative power domains, and the independence of the external forces with respect to the primary system dynamics will be ubiquitous in STA implementations and thus essential elements to evaluate actual energy consumptions. Whereas for slow processes the energy dissipated by friction can be made negligible (a standard assumption for infinite-time processes), even if the friction coefficient is not zero, STAs are, by definition, fast processes, so to neglect energy dissipation in STAs the stronger assumption of zero-friction coefficients is necessary. Again, the fast nature of STA protocols implies large positive and negative powers, which enhances the importance of braking. Braking mechanisms determine the cost of the energy integrated in negative power segments and if this energy can indeed be reused. In typical scenarios this is not the case, i.e., $\eta \neq 1$, so negative power segments consume energy (the extreme case is $\eta=-1$ ), or if they do not consume energy $(\eta=0)$, they do not compensate for the consumption in positive segments. For the realistic expectation that $\gamma \neq 0$ 
and $\eta \neq 1$, shorter process times imply higher-power peaks and an increased energy consumption. Note that even in the highly idealized limit $\gamma=0, \eta=1$, with zero global cost (with respect to final adiabatic energy minus initial energy), shortening the time also implies higher-power peaks, which become a limiting factor that cannot be ignored when determining the feasibility of a shortcut.

\section{ACKNOWLEDGMENTS}

We thank D. Guéry-Odelin and A. Levy for discussions. We acknowledge funding from the Basque government (Grant No. IT986-16), MINECO/FEDER, UE (Grants No. FIS201567161-P and No. FIS2015-70856-P), and QUITEMAD+CM S2013-ICE2801.
[1] X. Chen, A. Ruschhaupt, S. Schmidt, A. del Campo, D. GuéryOdelin, and J. G. Muga, Phys. Rev. Lett. 104, 063002 (2010).

[2] E. Torrontegui, S. Ibáñez, S. Martínez-Garaot, M. Modugno, A. del Campo, D. Guéry-Odelin, A. Ruschhaupt, X. Chen, and J. G. Muga, Adv. At. Mol. Opt. Phys. 62, 117 (2013).

[3] C. Jarzynski, Phys. Rev. A 88, 040101(R) (2013).

[4] M. Okuyama and K. Takahashi, J. Phys. Soc. Jpn. 86, 043002 (2017).

[5] C. Jarzynski, S. Deffner, A. Patra, and Y. Subaşı, Phys. Rev. E 95, 032122 (2017).

[6] M. Demirplak and S. Rice, J. Chem. Phys. 129, 154111 (2008).

[7] X. Chen and J. G. Muga, Phys. Rev. A 82, 053403 (2010).

[8] A. del Campo, J. Goold, and M. Paternostro, Sci. Rep. 4, 6208 (2014).

[9] A. C. Santos, R. D. Silva, and M. S. Sarandy, Phys. Rev. A 93, 012311 (2016).

[10] I. B. Coulamy, A. C. Santos, I. Hen, and M. S. Sarandy, Front. ICT 3, 19 (2016).

[11] Y. Zheng, S. Campbell, G. De Chiara, and D. Poletti, Phys. Rev. A 94, 042132 (2016).

[12] O. Abah and E. Lutz, Europhys. Lett. 118, 40005 (2017).

[13] K. Funo, J. N. Zhang, C. Chatou, K. Kim, M. Ueda, and A. del Campo, Phys. Rev. Lett. 118, 100602 (2017).

[14] R. Kosloff and Y. Rezek, Entropy 19, 136 (2017).

[15] M. Kieferová and N. Wiebe, New J. Phys. 16, 123034 (2014).

[16] S. Campbell and S. Deffner, Phys. Rev. Lett. 118, 100601 (2017).

[17] A. Bravetti and D. Tapias, arXiv:1706.07443.

[18] O. Abah and E. Lutz, arXiv:1707.09963.

[19] D. A. Winter, Biomechanics and Motor Control of Human Movement (Wiley, Hoboken, NJ, 2009).

[20] Z. Wu and X. Xia, IET Control Theory Appl. 8, 1833 (2014).

[21] X. Chen, E. Torrontegui, D. Stefanatos, J.-S. Li, and J. G. Muga, Phys. Rev. A 84, 043415 (2011).
[22] S. Masuda and K. Nakamura, Proc. R. Soc. A 466, 1135 (2010).

[23] E. Torrontegui, S. Ibáñez, X. Chen, A. Ruschhaupt, D. GuéryOdelin, and J. G. Muga, Phys. Rev. A 83, 013415 (2011).

[24] H. Goldstein, C. Poole, and J. Safko, Classical Mechanics, 3rd ed. (Adison-Wesley, Reading, MA, 2002).

[25] L. Meirovitch, Methods of Analytical Dynamics (McGraw-Hill, New York, 1970).

[26] S. Montgomery-Smith, Electron. J. Differ. Equations 2014, 89 (2014).

[27] H. R. Lewis and P. G. L. Leach, J. Math. Phys. 23, 2371 (1982).

[28] R. Landauer, Appl. Phys. Lett. 51, 2056 (1987).

[29] Y.-Y. Cui, X. Chen, and J. G. Muga, J. Phys. Chem. A 120, 2962 (2015).

[30] D. Stefanatos, J. Ruths, and J.-S. Li, Phys. Rev. A 82, 063422 (2010).

[31] L. S. Pontryagin, V. G. Boltyanskii, R. V. Gamkrelidze, and E. F. Mishchenko, The Mathematical Theory of Optimal Processes (Interscience, New York, 1962).

[32] D. Stefanatos and J.-S. Li, American Control Conf. 85, 5061 (2012)

[33] A. Couvert, T. Kawalec, G. Reinaudi, and D. Guéry-Odelin, Europhys. Lett. 83, 13001 (2008).

[34] R. Bowler, J. Gaebler, Y. Lin, T. R. Tan, D. Hanneke, J. D. Jost, J. P. Home, D. Leibfried, and D. J. Wineland, Phys. Rev. Lett. 109, 080502 (2012).

[35] A. Walther, F. Ziesel, T. Ruster, S. T. Dawkins, K. Ott, M. Hettrich, K. Singer, F. Schmidt-Kaler, and U. Poschinger, Phys. Rev. Lett. 109, 080501 (2012).

[36] A. Zenesini, H. Lignier, D. Ciampini, O. Morsch, and E. Arimondo, Phys. Rev. Lett. 102, 100403 (2009).

[37] A. Ruschhaupt, X. Chen, D. Alonso, and J. G. Muga, New J. Phys. 14, 093040 (2012). 